March, 1998

DFUB-98-04

\title{
TRUNCATED CONFORMAL SPACE AT $c=1$, NONLINEAR INTEGRAL EQUATION AND QUANTIZATION RULES FOR MULTI-SOLITON STATES
}

\author{
G. Feverati, F. Ravanini and G. Takács \\ Sez. INFN and Dip. di Fisica - Univ. di Bologna \\ Via Irnerio 46, I-40126 BOLOGNA, Italy
}

\begin{abstract}
We develop Truncated Conformal Space (TCS) technique for perturbations of $c=1$ Conformal Field Theories. We use it to give the first numerical evidence of the validity of the non-linear integral equation (NLIE) derived from light-cone lattice regularization at intermediate scales. A controversy on the quantization of Bethe states is solved by this numerical comparison and by using the locality principle at the ultraviolet fixed point. It turns out that the correct quantization for pure hole states is the one with half-integer quantum numbers originally proposed by Fioravanti et al. [8]. Once the correct rule is imposed, the agreement between TCS and NLIE for pure hole states turns out to be impressive.
\end{abstract}

\section{Introduction}

The scaling functions of $1+1$ dimensional integrable models on the cylinder have proven to be a very useful non-perturbative tool of investigation of their finite size effects, in particular of the renormalization flow properties of the vacuum as well as of the excited states. Various methods have been proposed for the calculation of these important quantities, as the Truncated Conformal Space (TCS) method [1] or the Thermodynamic Bethe Ansatz (TBA) [2, 3]. A very promising method is the one introduced some years ago by Destri and de Vega 四, 司 (similar methods were independently introduced in Condensed Matter Physics by other authors [6]). It consists in defining a lattice model and give evidence that its continuum limit reproduces the sine-Gordon QFT ( $\mathrm{sG}$ ) [0], then deriving a non-linear integral equation (NLIE) which is basically the continuum limit of the Bethe equations. There is one such equation for each excitation in the spectrum of the physical states. The problem is to give a general rule to write down the NLIE corresponding to a state characterized by a certain distribution of Bethe roots and holes. This problem, namely to find the NLIE for excited states, was first addressed, in a QFT context, in [8] which discussed the case of pure hole excitations. The general setup of excited states was described in detail later in [9]. The solution of the NLIE, the so-called counting function, is a central object in Bethe Ansatz approach to integrable QFT from which it is possible to reconstruct the eigenvalues of all the local conserved currents of the theory put on a cylinder of circumference $L$. 
The aim of this paper is to present a numerical comparison between the NLIE and TCS. We develop a TCS for $c=1$ CFT perturbed by its $\cos \beta \phi$ operator, that defines the sG model as perturbation of a CFT. We compare TCS data against scaling functions computed from numerical integration of NLIE and find a very good agreement, especially in the attractive regime, but also in the repulsive one. This agreement, however, only shows up for a specific choice of the quantization rule for the Bethe Ansatz states which is different from the one reported in [9] and instead agrees with the one made in ref. [8]. This leads to the observation that the NLIE produces in fact more states than those present in the sG Hilbert space. It is known that the relation among the Hilbert space of $c=1 \mathrm{CFT}$, the sG model and the massive Thirring (mTh) model is a quite delicate issue [10]. It is out of the scope of the present letter to investigate this very important question. We intend to return to it in a more extensive paper where a more detailed analysis of the results presented here will be given [11]. It is important to keep in mind that the NLIE (and in general Bethe Ansatz methods) are only able, at present, to reproduce states with even topological charge (i.e. even number of solitons minus antisolitons) in $\mathrm{sG}$ model.

\section{The NLIE and its properties}

The Lagrangian of sG theory is

$$
\mathcal{L}=\int d x\left(\frac{1}{2} \partial_{\mu} \Phi \partial^{\mu} \Phi+\frac{\mathcal{M}^{2}}{\beta^{2}}: \cos (\beta \Phi):\right)
$$

Often it is convenient to use the parameter $p=\frac{\beta^{2}}{8 \pi-\beta^{2}}$ or the 6 -vertex anisotropy $\gamma=\frac{\pi}{p+1}$. 1

We do not reproduce here the deduction of the NLIE from the lattice Bethe equations. Although there are subtle remarks to do on all the derivations presented so far [5, 9], this aspect is out of the scope of this short letter and will be dealt with in a forthcoming longer publication [11. Here we are content to present the final equation and explain how it can be used to investigate the finite size corrections to energy-momentum of the excited states.

The NLIE for the $\mathrm{sG} / \mathrm{mTh}$ model reads

$$
Z(\lambda)=l \sinh \lambda+g\left(\lambda \mid \lambda_{j}\right)+2 \Im \mathrm{m} \int_{-\infty}^{+\infty} d x G(\lambda-x-i \eta) \log \left(1+(-1)^{\delta} e^{i Z(x+i \eta)}\right)
$$

where $\eta$ is a positive free parameter, small enough not to reach any singularity of the counting function $Z$ off the real axis. With such prescription, eq. (11) is totally independent of the choice of $\eta$ by Cauchy's theorem. $l$ is related to the circumference of the space-time cylinder $L$ (the spatial volume) by $l=M L$, where $M$ is the mass scale of the theory, chosen to be equal to the soliton mass. The kernel $G(\lambda)$ is given by

$$
G(\lambda)=\int_{-\infty}^{+\infty} d k e^{i k \lambda} \frac{\sinh \frac{(p-1) k \pi}{2}}{\sinh \frac{p k \pi}{2} \cosh \frac{k \pi}{2}} .
$$

${ }^{1}$ In terms of $p, p=1$ is the free fermion point, and $p=\frac{1}{k}, k=1,2, \ldots$ are the thresholds where a new breather appears. $p<1$ corresponds to the attractive and $p>1$ to the repulsive regime. 
The source term $g\left(\lambda \mid \lambda_{j}\right)$ is specific to the state considered and vanishes in the case of the vacuum, which in Bethe Ansatz language corresponds to a sea of real roots.

The counting function $Z(\lambda)$ has the property that any Bethe root satisfies

$$
e^{i Z\left(\lambda_{j}\right)}=-(-1)^{\delta}
$$

The converse is not true, i.e. there are values $\lambda_{h}$ for which $e^{i Z\left(\lambda_{h}\right)}=-(-1)^{\delta}$ that are not solutions of the Bethe equations. Such locations on the real axis are called holes.

Excited states are seen, in a typical Bethe Ansatz spirit, as different root configurations for a system of Bethe Ansatz equations and are given by creating some holes in the vacuum sea and adding some complex roots. Holes and complex roots are $O(1)$ in number, while the real roots of the vacuum sea are $O(N)$ ( $N$ being the number of sites), and tend to infinity in the continuum limit. The number $\delta$ is equal to the number of roots modulo 2 on the lattice.

For a state having $H$ holes at positions $\lambda_{h}$ on the real axis, $N_{c}$ complex roots $\lambda_{c}$ (of close type) such that $\left|\Im \mathrm{m} \lambda_{c}\right|<\pi \min (1, p), N_{w}$ complex roots (of wide type) with $\left|\Im \mathrm{m} \lambda_{c}\right| \geq \pi \min (1, p)$ and $N_{s}$ special roots/holes $\lambda_{s}$ :

$$
g\left(\lambda \mid \lambda_{j}\right)=\sum_{h=1}^{H} \chi\left(\lambda-\lambda_{h}\right)-\sum_{c=1}^{N_{c}} \chi\left(\lambda-\lambda_{c}\right)-\sum_{w=1}^{N_{w}} \chi_{I I}\left(\lambda-\lambda_{w}\right)-2 \sum_{s=1}^{N_{s}} \chi\left(\lambda-\lambda_{s}\right)
$$

where $\lambda_{j}$ denotes the set of all parameters $(j=h, c, w, s)$. The function $\chi(\lambda)$ is the odd primitive of $G(\lambda)$ and

$$
\chi_{I I}(\lambda)=\chi(\lambda)+\operatorname{sign}(p-1) \chi(\lambda-i \pi \min (1, p) \operatorname{sign}(\Im m \lambda)) .
$$

is the so-called second determination of the function $\chi$. The interested reader can find more details in [9].

The parameters $\lambda_{j}$ are determined by the Bethe conditions

$$
Z\left(\lambda_{j}\right)=2 \pi I_{j} \quad, \quad I_{j} \in \mathbf{Z}+\frac{1+\delta}{2}
$$

which are the continuum analogs of the logarithms of the Bethe equations. Any root of the Bethe equations, as well as any hole, satisfies (3) and its position is therefore fixed by giving its Bethe quantum number $I_{j}$ which is half-integer for $\delta=0$ and integer for $\delta=1$.

The number $N_{s}$ of special roots/holes $\lambda_{s}$, for which $Z^{\prime}\left(\lambda_{s}\right)<0$, as introduced in [9], need not to be specified a priori for a state. Indeed one can easily convince oneself that in the deep infrared limit $l \rightarrow \infty, Z^{\prime}(\lambda)$ is always positive and these objects never appear. Their appearance in some states for values of $l$ smaller than a certain $l_{0}$ is dictated by the fact that sometimes the logarithm in the convolution term of the equation fails to remain in its fundamental domain, and an analytic continuation imposes to modify the equation itself by adding new terms to the source. The number $H_{e f f}=H-2 N_{s}$ is invariant for a given state as the spatial volume $l$ varies and coincides with the number of dressed solitonic particles in the state.

The number of the various species of roots and holes in a configuration is not arbitrary but rather is subject to constraints originating from an analysis of the asymptotics of the lattice counting function. These constraints can be recast in a form that does not contain the number 
of real roots, and therefore their validity can be extended to the continuum limit, in a form that we call the counting equation [9]

$$
H_{\text {eff }}=2 S+M_{c}+2 M_{w} \Theta(p-1)
$$

where $\Theta(x)$ denotes the usual step function and $S$ is the total spin of the Bethe Ansatz system, which can be identified with half of the sG topological charge $Q$.

Once the function $Z(\lambda)$ is evaluated as a solution of the NLIE, it can be used to compute the energy-momentum $(E, P)$ (or in principle the eigenvalue of any other local integral of motion)

$$
P^{ \pm}=E \pm P=z\left(\lambda \mid \lambda_{j}\right)-2 M \Im \mathrm{m} \int_{-\infty}^{+\infty} \frac{d \lambda}{2 \pi} e^{ \pm \lambda} \log \left(1+(-1)^{\delta} e^{i Z(\lambda+i \eta)}\right)
$$

where

$$
z\left(\lambda \mid \lambda_{j}\right)=M\left(\sum_{h=1}^{N_{h}} e^{ \pm \lambda_{h}}-\sum_{c=1}^{N_{c}} e^{ \pm \lambda_{c}}+\Theta(1-p) \sum_{w} e^{ \pm \lambda_{w}}\left(1+e^{\mp i \pi(p+1) \operatorname{sign}\left(\Im m \lambda_{w}\right)}\right)-2 \sum_{s=1}^{N_{s}} e^{ \pm \lambda_{s}}\right)
$$

In particular, $P^{ \pm}(L)$ for $L \rightarrow \infty$ reproduces the asymptotic particle content of the state. For $L \rightarrow 0$ instead, it is expected to be related to the central charge $c$ and conformal dimensions $\Delta^{ \pm}$of the ultraviolet CFT state by $P^{ \pm}=-\frac{\pi}{6 L}\left(c-24 \Delta^{ \pm}\right)$.

Notice that eq. (11) does not coincide with the one reported in [9. The argument of one of logarithmic terms in $\| 9$ differs by a phase $(-1)^{\delta}$. Our choice gives the correct infrared asymptotic $O\left(e^{-l}\right)$ for the IR asymptotics of finite size effects, as it must be in a massive theory. The expression in [9] would instead give a rapidly oscillating integrand, which gives a contribution that can be (after tedious calculations) proven to be only $O(1 / l)$ in the IR limit. A careful analysis of logarithmic branches confirms that our choice is the correct one. We shall report the details about this point in a later publication 11 .

\section{$3 \quad$ UV behaviour of multi-soliton states}

We consider sG theory as the perturbation of a $c=1$ massless free boson $\Phi$ compactified on circle of radius $R$ by a potential $V=\frac{g}{2}:\left(e^{i \beta \Phi}+e^{-i \beta \Phi}\right):$, where $g=\frac{\mathcal{M}^{2}}{\beta^{2}}$. The interaction term has conformal dimensions $\Delta_{V}=\Delta_{V}^{ \pm}=\frac{\beta^{2}}{8 \pi}=\frac{p}{p+1}$ and becomes marginal when $\beta^{2}=8 \pi$ which corresponds to $p=\infty$. With the convention adopted here, the primary states of the unperturbed CFT, have left and right conformal dimensions

$$
\Delta_{n, m}^{ \pm}=\frac{1}{2}\left(\frac{n}{R} \pm \frac{1}{2} m R\right)^{2}=\frac{n^{2}}{2 R^{2}}+\frac{m^{2} R^{2}}{8} \pm \frac{n m}{2}
$$

They are created by the vertex operators $V_{n, m}$, where $n \in \mathbf{R}$ and the winding number $m$ (which is the eigenvalue of the topological charge $Q$ ) satisfies $m \in \mathbf{Z}$. Locality of the operator product

${ }^{2}$ Note that this convention is different from the one usually adopted in the literature (see $\| 10$ and references therein), where the compactification radius is $1 / R$ and the elementary sine-Gordon field is the dual of the conformal scalar at the UV fixed point. In our convention, the conformal scalar field is the UV limit of the elementary sine-Gordon field. 
algebra puts a severe constraint on the operator content of the UV field theory. At a generic value of the coupling constant $\beta$ there are only two different possible maximal local subalgebras of the algebra generated by the vertex operators [10], namely a bosonic algebra $A_{b}$ generated by operators with $\{n, m \in \mathbf{Z}\}$ which corresponds to the modular invariant partition function and is the UV limit of the $\mathrm{sG}$ model, and a fermionic algebra $A_{f}$ generated by operators with $\{n \in \mathbf{Z}, m \in 2 \mathbf{Z}\} \bigcup\left\{n \in \mathbf{Z}+\frac{1}{2}, m \in 2 \mathbf{Z}+1\right\}$ corresponding to the $\Gamma_{2}$ invariant partition function and the UV limit of mTh field theory. The two algebras overlap in the sector with even winding number (topological charge) which is the sector common in the two off-critical field theories and is the one that so far has been studied using the NLIE. We limit ourselves to this sector in this paper too.

The ultraviolet behaviour of the solutions of the NLIE can be obtained in the so-called " $k i n k$ limit" well-known from TBA. The method of calculation is the same as outlined in [8\||9] so let us just give the results. For the vacuum (quantized with half-integer Bethe quantum numbers) one gets the leading behaviour

$$
P^{ \pm}=-\frac{\pi c}{6 L}+\ldots, L \rightarrow 0
$$

with $c=1$, which is the correct UV central charge.

For a two-hole state quantized with integers (which is the prescription of [9]) and having a left-moving hole with quantum number $I^{+} \geq 0$ and a right-moving one with quantum number $I^{-} \leq 0\left(\right.$ with $\left.I^{+} \neq I^{-}\right)$we obtain

$$
\Delta^{ \pm}=\frac{1}{8 R^{2}}+\frac{1}{2} R^{2}-\frac{1}{2} \pm I^{ \pm}
$$

which corresponds to the vertex operators $V_{ \pm \frac{1}{2}, 2}$ and their descendants. As these operators are not included in any of the two possible maximal local operator algebras, we have to exclude the integer-quantized two-hole states. The UV dimensions of the same two-hole configuration with half-integer quantization turns out to be

$$
\Delta^{ \pm}=\frac{1}{2} R^{2}-\frac{1}{2} \pm I^{ \pm}
$$

which instead describes $V_{0,2}$ and its descendants, the primary state corresponding to the minimal choice $I^{ \pm}= \pm \frac{1}{2}$. This is in fact the vertex operator that one expects to be the UV limit of the lowest lying two-soliton state in view of the results in $[10$. The results presented in $\mid 9 \|$ are different when specialised to the two-hole states. Since (as shown in the next section) the numerical results confirm our calculations we think that the calculation of the ultraviolet dimensions in [9] should be taken with care.

For generic states with even number of solitons $H$, half of which move to the left and half to the right, with their quantum numbers not necessarily symmetrical to the origin, choosing half-integer quantization we obtained that the UV limit corresponds to the family $V_{0, H}$ (the primary state given by the lowest possible choice of Bethe quantum numbers). This contradicts the rule in [9] which is integer quantization for $H / 2$ odd and half-integer for $H / 2$ even, but is in accordance with what is expected from the UV conformal field theory and agrees with the numerical results we present in the next section. Integer quantization, instead, leads to the vertex operators $V_{ \pm \frac{1}{2}, H}$ and their descendants, which are again excluded by locality. 


\section{Truncated Conformal Space at $c=1$}

In this section we describe results obtained using the Truncated Conformal Space (TCS) method which support the validity of the NLIE describing the vacuum and pure hole states.

The TCS method was originally created to describe perturbations of Virasoro minimal models in finite spatial volume [1]. We have developed an extension of the method to study perturbations of a $c=1$ compactified boson, more closely the perturbation corresponding to $\mathrm{sG}$ theory.

The perturbation keeps the winding number $m$ and the momentum $P$ conserved. Therefore, the Hilbert space of the massive theory can be split into sectors labelled by the values of $P$ and $m$, which are quantized by integers. The TCS method consists of retaining only those states in such a sector for which the eigenvalue of the UV conformal Hamiltonian $H_{C F T}$ is less than a certain upper value $E_{c u t}$, so the truncated space is defined as

$$
\mathcal{H}_{T C S}\left(s, m, E_{\text {cut }}\right)=\left\{|\Psi\rangle: P|\Psi\rangle=s|\Psi\rangle, Q|\Psi\rangle=m|\Psi\rangle, H_{C F T}|\Psi\rangle \leq E_{\text {cut }}|\Psi\rangle\right\} .
$$

For a given value of $s, m$ and $E_{c u t}$ this space is always finite dimensional. In this space, the Hamiltonian is represented by a finite size matrix whose explicit form is:

$$
\widehat{H}=\frac{2 \pi}{L}\left(\widehat{L_{0}}+\widehat{\bar{L}_{0}}-\frac{c}{12} \widehat{I}+g \frac{L^{2-2 \Delta_{V}}}{(2 \pi)^{1-2 \Delta_{V}}} \widehat{B}\right)
$$

where $\widehat{L_{0}}$ and $\widehat{\overline{L_{0}}}$ are diagonal matrices with their diagonal elements being the left and right conformal weights, $\widehat{I}$ is the identity matrix and the matrix elements of $\widehat{B}$ are

$$
\widehat{B}_{\Phi, \Psi}=\langle\Phi|: \cos (\hat{\beta} \varphi(1,1)):| \Psi\rangle
$$

We choose our energy units in terms of the soliton mass $M$, related to the coupling constant $g$ by the mass gap formula given in ref. [12]:

$$
g=\kappa M^{2-2 \Delta_{V}} \quad, \quad \kappa=\frac{2 \Gamma\left(\Delta_{V}\right)}{\pi \Gamma\left(1-\Delta_{V}\right)}\left(\frac{\sqrt{\pi}}{2 \Gamma\left(\frac{1}{2-2 \Delta_{V}}\right) \Gamma\left(\frac{h}{4-4 \Delta_{V}}\right)}\right)^{2-2 \Delta_{V}} .
$$

The TCS method provides a nonperturbative way to obtain the spectrum (the mass gap, the mass ratios and the scattering amplitudes) numerically which can serve as a tool to check the predicted exact results of integrable field theories and get a picture of the physical behaviour even for the non-integrable case. The systematic error introduced by the truncation procedure is called the truncation error. It increases with the volume $L$ and can be made smaller by increasing the truncation level (at the price of increasing the size of the matrices, which is bound from above by machine memory and computation time). 
While we do not describe technical details of the TCS computation (we refer the interested reader to [1]), we do have to make some remarks on how the TCS method applies to $c=1$ theories.

The (dimensionless) energy of any state $\Psi$ (the scaling function) goes with the volume $L$ as

$$
\frac{E_{\Psi}(L)}{M}=-\frac{\pi\left(c-12\left(\Delta_{\Psi}^{+}+\Delta_{\Psi}^{-}\right)\right)}{6 l}+B l+\sum_{k=1}^{\infty} C_{k}(\Psi) l^{k\left(2-2 \Delta_{V}\right)},
$$

where $\Delta_{\Psi}^{ \pm}$are the left/right conformal dimensions of the state in the ultraviolet limit, $B$ is the universal bulk energy constant (the vacuum energy density) and the infinite sum represents the perturbative contributions from the potential $V$.

The bulk energy constant has been predicted from TBA and reads [12]

$$
B=-\frac{1}{4} \tan \left(\frac{p \pi}{2}\right)
$$

(the same was obtained from the NLIE in [5]). This is a highly non-analytic function of $p$ and it becomes infinite at the points where $p$ is an odd integer. In fact, at these points there is a value of $k$ for which $k\left(2-2 \Delta_{V}\right)=2$, and $C_{k}(\Psi) \rightarrow \infty$. The infinite parts of $B$ and $C_{k}(\Psi)$ exactly cancel, leaving a logarithmic (proportional to $l \log l$ ) and a finite linear contribution to the energy, by a sort of a resonance mechanism.

In the attractive regime the results of TCS converge very well when increasing the truncation level $E_{c u t}$ (as far as we do not approach too much the free fermion point $p=1$ ). On the contrary, in the repulsive regime there are convergence problems for the scaling functions $E_{\Psi}(L)$. These are related to certain ultraviolet divergences in conformal perturbation theory already known in the literature (for a detailed discussion see [13]), which disappear when we subtract the vacuum energy from the excited states energies (which is a sort of a renormalization procedure). The relative energies $\mathcal{E}_{\Psi}(L)=E_{\Psi}(L)-E_{\text {vac }}(L)$ converge well, which can be seen e.g. from the fact that the mass gap acquires its exact value (which is 1 in our units) quite rapidly when increasing the truncation level. Consequently, in the repulsive regime one can only trust the relative scaling functions produced by the TCS method, while in the attractive regime we will see that even the absolute energy values agree very well with the predictions of the NLIE (including the predicted bulk energy constant (5), which is completely analytic for $p<1$ and thus logarithmic corrections are absent as well).

Even in the attractive regime, to get reliable results we had to work with dimensions around 4000 , which was just around the limitation of our machines. Diagonalization for one sector took around one day for twenty evenly spaced values of the volume $L$. This shows that the TCS for $c=1$ theories is far less convergent than the one for minimal models (in the original Yang-Lee example the authors of [1] took a 17 dimensional Hilbert space (!) and arrived to very accurate results). Such a slow convergence meant that the total procedure (building up the basis, calculating the matrix elements and diagonalizing the Hamiltonian) had to be done completely in a compiled $\mathrm{C}$ program. For the matrix diagonalization, we used the LAPACK numerical linear algebra library, freely available from Netlib over the Internet. 


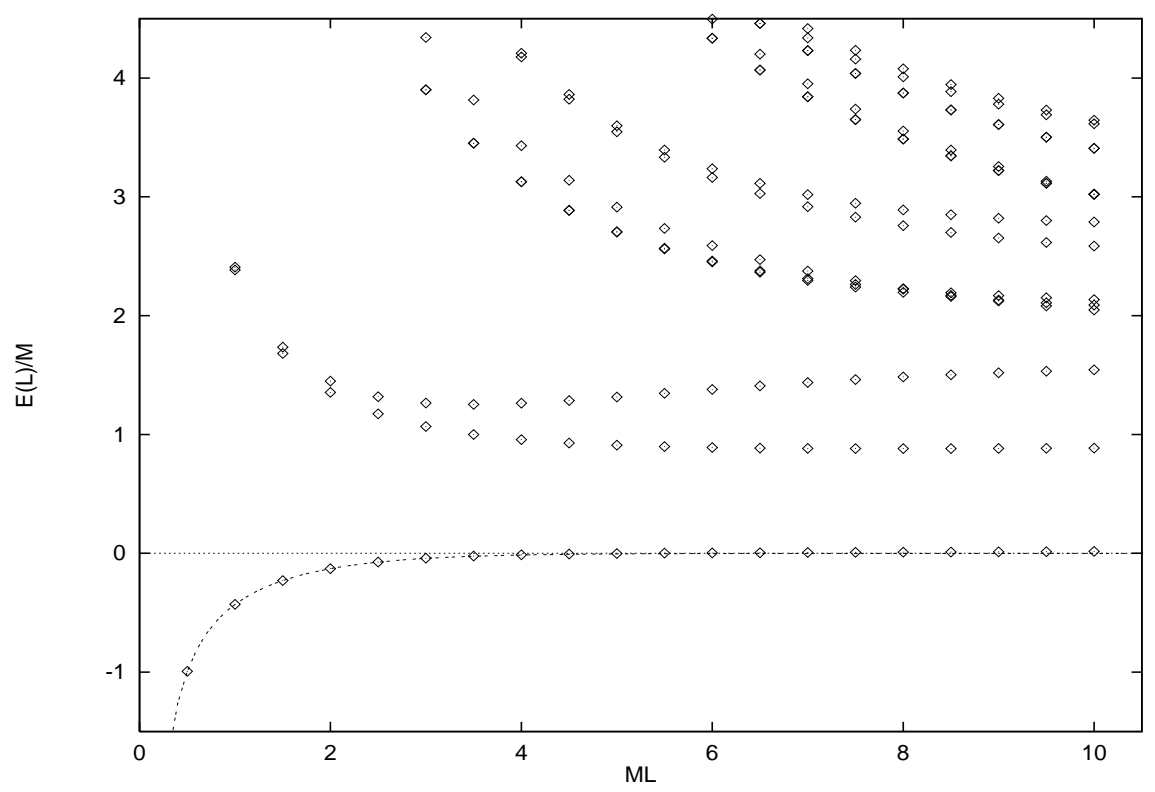

Figure 1: The first few energy levels in the vacuum $(m=0)$ sector at $p=\frac{2}{7}$ (plotted with diamonds) for $E_{c u t}=17.0$ (the dimension of the space is 4141) and the NLIE prediction for the vacuum scaling function (shown with a continuous line).

\section{$5 \quad$ Numerical results}

\subsection{TCS and NLIE in the attractive regime}

We have made the comparison of the numerical data from TCS and the NLIE predictions at several values of the parameter $p$. For illustration, we present the case of $p=\frac{2}{7}$. For this and all other values of the coupling constant, we obtained a spectacular agreement between the results obtained by the two methods, up to deviations of order $10^{-4}-10^{-3}$ which are smaller than the size of the points in the plots. The deviation grows with the volume $L$, exactly as expected for truncation errors. By studying other parameters such as the mass gap, the breather-soliton mass ratios and the rate of convergence of the energy levels with increasing the value of $E_{c u t}$, the small differences can be clearly attributed to the inaccuracy in the TCS data.

Figures 1, 2 and 3 show the results in the $m=0,2$ and 4 sectors, respectively. The value of the predicted bulk energy (5) has been subtracted from TCS data in order to normalize them the same way as the NLIE data are. Note that in order to achieve agreement the holes should be quantized with half-integers. We have made some trials of integer quantization in the NLIE, but the results obtained differed significantly from the TCS data, with errors of the order $10 \%$.

\subsection{The repulsive regime}

In the repulsive regime the absolute scaling functions do not converge well in the TCS method as it was described in Section 4 above. We take as an example the value of the coupling $p=1.5$ and plot the relative scaling functions obtained from TCS, comparing them with the corresponding ones obtained from the NLIE (figures 4 and 5). One can see that the truncation errors become 


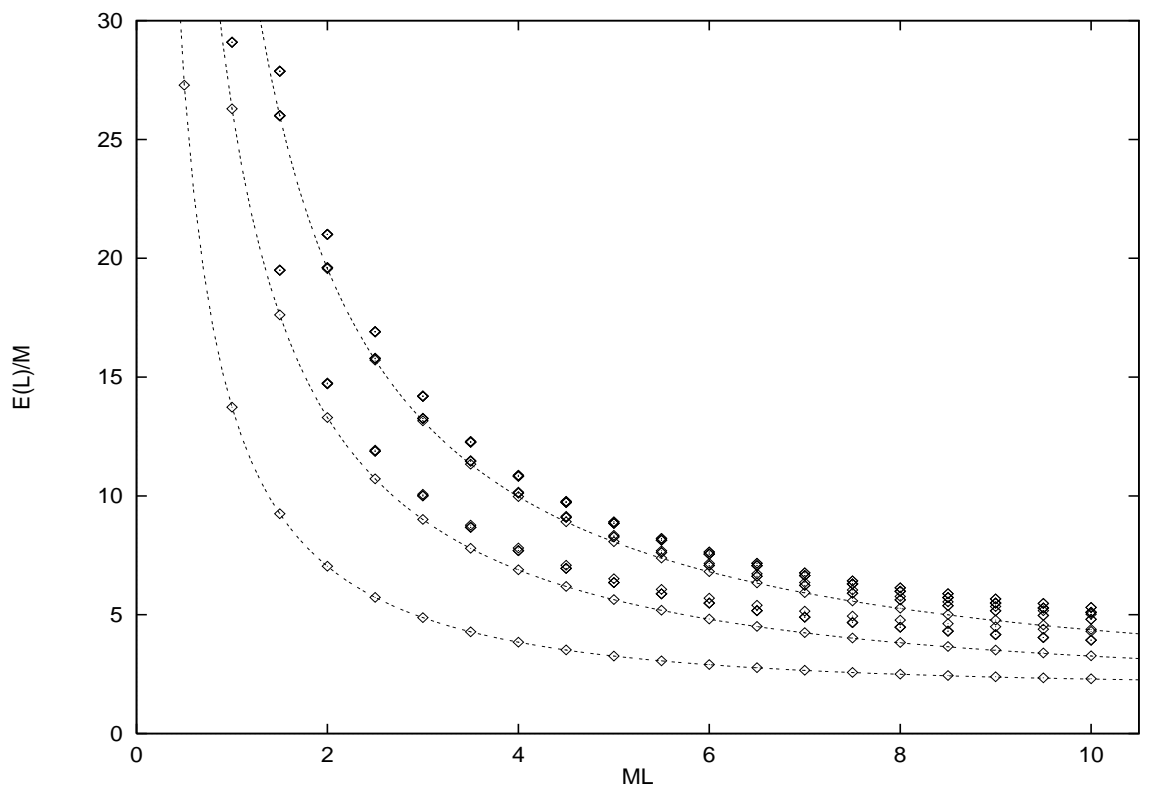

Figure 2: The first few energy levels in the $m=2$ sector at $p=\frac{2}{7}$ (plotted with diamonds) for $E_{c u t}=20.0$ (the dimension of the space is 3917) and the NLIE prediction for the two hole scaling functions with quantum numbers $\left(-\frac{1}{2}, \frac{1}{2}\right),\left(-\frac{3}{2}, \frac{3}{2}\right)$ and $\left(-\frac{5}{2}, \frac{5}{2}\right)$ (shown with continuous lines).

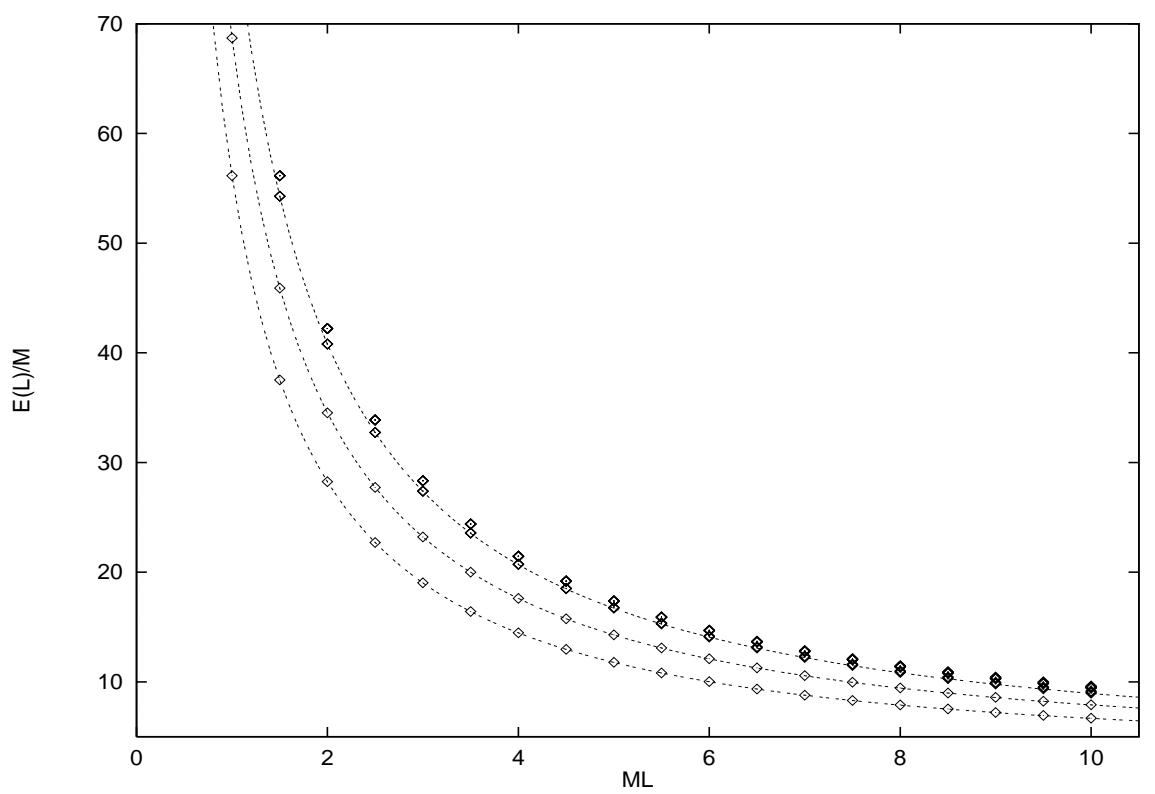

Figure 3: The first few energy levels in the $m=4$ sector at $p=\frac{2}{7}$ (plotted with diamonds) for $E_{c u t}=26.0$ (the dimension of the space is 3403) and the NLIE prediction for the four hole scaling functions with quantum numbers $\left(-\frac{3}{2},-\frac{1}{2}, \frac{1}{2}, \frac{3}{2}\right),\left(-\frac{5}{2},-\frac{1}{2}, \frac{1}{2}, \frac{5}{2}\right)$ and $\left(-\frac{5}{2},-\frac{3}{2}, \frac{3}{2}, \frac{5}{2}\right)$ (shown with continuous lines). 


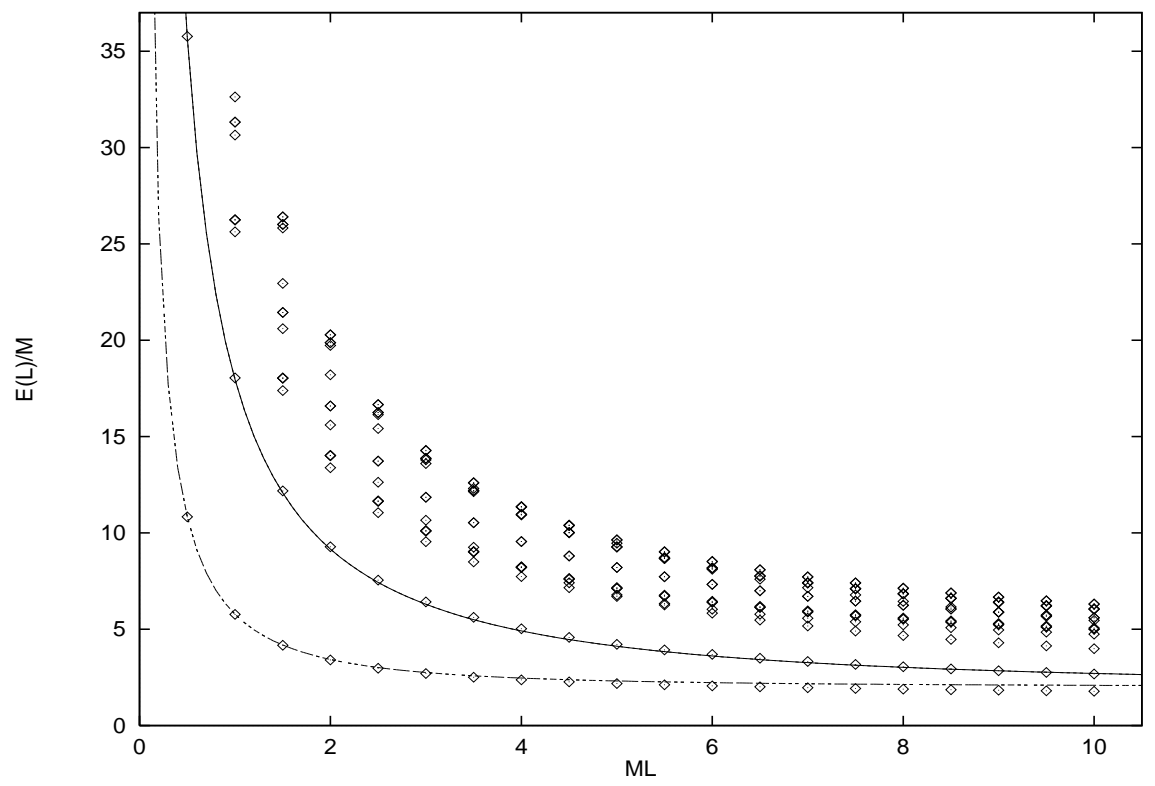

Figure 4: The first few energy levels relative to the vacuum in the $m=2$ sector at $p=1.5$ (plotted with diamonds) for $E_{\text {cut }}=20.0$ (the dimension of the space is 4445 ) and the NLIE prediction for the relative two hole scaling functions with quantum numbers $\left(-\frac{1}{2}, \frac{1}{2}\right)$ and $\left(-\frac{3}{2}, \frac{3}{2}\right)$ (shown with continuous lines).

larger, at values of the volume $l$ close to 10 the deviation can be observed even from the figures. For $l<5$ the agreement is still within an error of order $10^{-3}$. Once again, results obtained with integer quantization for the holes showed a large deviation from the TCS data.

\section{Conclusions}

This letter should be seen as a preliminary report of the work done by our group to better investigate and clarify some of the properties of the NLIE in sG theory. We have given the first numerical check against Truncated Conformal Space data - the only checks so far have been calculations of the conformal spectrum at small scale (kink limit) and comparison with the Factorized Scattering Theory at large scale. The results presented here are the first evidence of the validity of NLIE in the intermediate range of scales and thus show that the NLIE describes correctly the interpolation between the UV and IR asymptotic regions. Our approach has also resolved some ambiguities about the choice of quantization rule for the Bethe quantum numbers: pure hole states are always quantized by half-integers in the sG model on a cylinder with periodic boundary conditions. Work is in progress to extend the results to complex roots, the outcome of which will be published - together with details omitted in this paper - in a forthcoming article [11.

Acknowledgements - We are indebted to C. Destri, V. A. Fateev and E. Quattrini for useful discussions. This work was supported in part by NATO Grant CRG 950751, by European Union TMR Network FMRX-CT96-0012 and by INFN Iniziativa Specifica TO12. G. T. has 


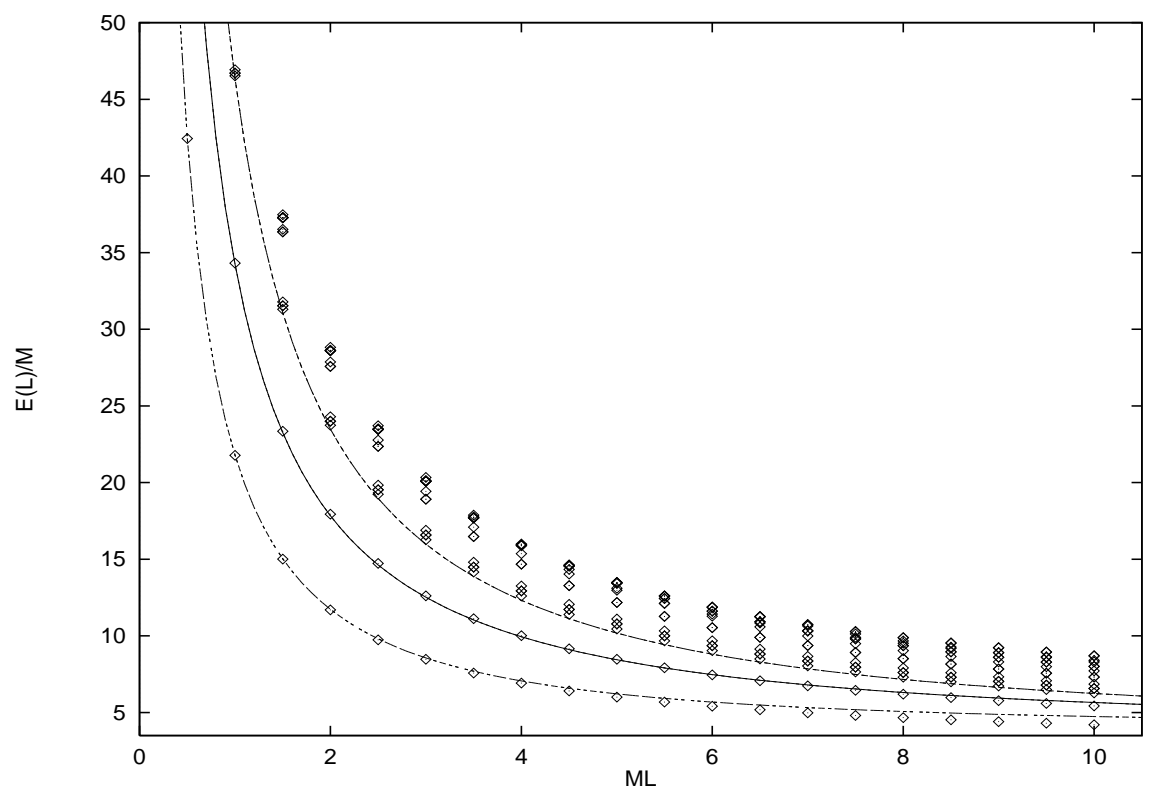

Figure 5: The first few energy levels relative to the vacuum in the $m=4$ sector at $p=1.5$ (plotted with diamonds) for $E_{\text {cut }}=22.5$ (the dimension of the space is 4149) and the NLIE prediction for the relative two hole scaling functions with quantum numbers $\left(-\frac{3}{2},-\frac{1}{2}, \frac{1}{2}, \frac{3}{2}\right)$, $\left(-\frac{5}{2},-\frac{1}{2}, \frac{1}{2}, \frac{5}{2}\right)$ and $\left(-\frac{5}{2},-\frac{3}{2}, \frac{3}{2}, \frac{5}{2}\right)$ (shown with continuous lines).

been partially supported by the FKFP 0125/1997 and OTKA T016251 Hungarian funds.

\section{References}

[1] V.P. Yurov and A.B. Zamolodchikov, Int.J.Mod.Phys. A5 (1990) 3221-3246.

[2] C.N. Yang and C.P. Yang, J. Math. Phys. 10 (1969) 1115.

[3] Al.B. Zamolodchikov, Nucl. Phys. B342 (1990) 695-720.

[4] C. Destri and H.J. De Vega, Phys. Rev. Lett. 69 (1992) 2313-2317.

[5] C. Destri and H.J. De Vega, Nucl. Phys. B438 (1995) 413-454, hep-th/9407117.

[6] A. Klümper and P.A. Pearce, J. Stat. Phys. 64 (1991) 13;

A. Klümper, M. Batchelor and P.A. Pearce, J. Phys. A23 (1991) 3111.

[7] C. Destri and H.J. De Vega, Nucl. Phys. B290 (1987) 363-391.

[8] D. Fioravanti, A. Mariottini, E. Quattrini and F. Ravanini, Phys. Lett. B390 (1997) 243251, hep-th/9608091.

[9] C. Destri and H. De Vega, Nucl. Phys. B504 (1997) 621-664, hep-th/9701107.

[10] T. Klassen and E. Melzer, Int. J. Mod. Phys. A8 (1993) 4131-4174, hep-th/9206114. 
[11] G. Feverati, F. Ravanini and G. Takács, in preparation.

[12] Al.B. Zamolodchikov, Int. J. Mod. Phys. A10 (1995) 1125-1150.

[13] T.R. Klassen and E. Melzer, Nucl. Phys. B370 (1992) 511-550. 